\title{
BMJ Open Prediction of emotional exhaustion over the course of the COVID-19 pandemic in primary care dental staff: an intensive longitudinal study
}

Ruth Freeman, ${ }^{1}$ Jennifer Knights, ${ }^{1,2}$ Laura Beaton, ${ }^{2}$ Mariana Araujo, ${ }^{2}$ Siyang Yuan, ${ }^{3}$ Jan Clarkson, ${ }^{1,2}$ Linda Young, ${ }^{2}$ Gerald Humphris (i) 4

To cite: Freeman R, Knights J, Beaton L, et al. Prediction of emotional exhaustion over the course of the COVID-19 pandemic in primary care dental staff: an intensive longitudinal study. BMJ Open 2021;11:e054827. doi:10.1136/ bmjopen-2021-054827

- Prepublication history and additional supplemental material for this paper are available online. To view these files, please visit the journal online (http://dx.doi.org/10.1136/ bmjopen-2021-054827).

Professor Freeman died on 23rd September 2021.

Received 26 June 2021 Accepted 09 December 2021

D) Check for updates

(C) Author(s) (or their employer(s)) 2021. Re-use permitted under CC BY-NC. No commercial re-use. See rights and permissions. Published by BMJ.

${ }^{1}$ Dental Health Services Research Unit, University of Dundee, Dundee, UK ${ }^{2}$ NHS Education for Scotland, Edinburgh, UK

${ }^{3}$ School of Dentistry, University of Dundee, Dundee, UK

${ }^{4}$ Population and Behavioural Sciences, Medical School, University of St Andrews, St Andrews, UK

Correspondence to Professor Gerald Humphris; gmh4@st-andrews.ac.uk

\section{ABSTRACT}

Objective To investigate the fatigue trajectory during a 6-month duration of the COVID-19 pandemic for dental health professionals in primary dental care and for those in training. Design A longitudinal intensive panel diary was conducted (July-December 2020) assessing weekly selfratings of emotional fatigue.

Setting Dental care services in Scotland. Participants Dental trainees and primary dental care staff $(\mathrm{N}=53)$. These data were merged with respondents' replies to a cross-sectional baseline survey (Humphris et a). Recruitment was through 'Portal', an online tool administered by National Health Service Education for Scotland. Questback software was employed for data collection.

Primary and secondary outcome measures The diary questionnaire, consisted of a three-item fatigue scale and, from the baseline survey, three multi-item scales including: preparedness (14 items of the Dental Professional Preparedness for Practice Scale), the 22-item Impact of Event Scale-Revised and depressive symptomatology using the Patient Health Questionnaire-2. Statistical analysis included estimating the variability of fatigue over the study duration using a multilevel linear growth model. In addition, a path analysis was performed to determine the ability of preparedness and COVID-19 pandemic trauma to predict fatigue trajectories directly, or mediated through depressive symptomatology.

Results A large variation of fatigue trajectories $(p<0.001)$ was found from staff who completed a mean of 11 weekly uploads of their diary (diary uploads: minimum 4-maximum 24). The average slope was positive (standardised estimate $=0.13$, $95 \% \mathrm{Cl}: 0.07$ to $0.19, \mathrm{p}<0.001)$. Slope variation was modelled successfully from baseline data showing direct and indirect effects from preparedness, trauma and depressive symptoms. Conclusion Staff who volunteered to participate over a significant period of the COVID-19 pandemic in Scotland displayed a highly significant variety of individual trajectories. The results show some consistency with a job demands-resources model of burnout development. Suitable resource structures are indicated for staff over periods of extensive public service change.

\section{BACKGROUND}

The COVID-19 pandemic has created notable challenges to those delivering clinical care
Strengths and limitations of this study

- This is the first longitudinal intensive panel diary of dental trainees and primary dental care staff in Scotland.

- Study was conducted over a 6-month duration (July-December 2020) to assess weekly self-ratings of emotional fatigue.

- Data were merged with respondents' replies to a cross-sectional baseline survey that assessed preparedness, the trauma associated with COVID-19 (Impact of Event Scale-Revised) and depressive symptomatology (Patient Health Questionnaire-2).

- The variability of fatigue over the study duration was estimated, and a path analysis was performed to determine if preparedness and trauma predicted fatigue trajectories directly or mediated through depressive symptomatology.

- The dynamics of emotional fatigue were inspected solely using linear models and limited to 53 volunteer staff members.

within health services. A systematic review of hospital staff has demonstrated how direct contact with patients with COVID-19 predicted poorer levels of six mental health indicators. 1 Dental staff have arguably one of the most sensitive clinical environments to prepare for the delivery of care due to the close proximity with their patients and the nature of interventions to produce frequent conditions that threaten virus transmission, for instance through use of instruments that encourage airborne production of the virus. Our research group has reported a crosssectional survey of dental staff in primary care and trainees. ${ }^{2}$ The focus of the survey was to assess levels of preparedness of clinical staff in primary dental care for the pandemic as well as preparedness to deliver care, associated emotional trauma, burnout and depressive symptomatology. Results indicated a significant burden of burnout, especially emotional 
exhaustion, as well as depressive symptomatology. In addition, the degree of preparedness for delivery of services during the COVID-19 pandemic rated by the respondents predicted depressive symptoms negatively. The conclusions, although instructive, were limited due the cross-sectional study design. The authors strongly recommended the need for further work to determine the dynamic nature of the psychological response to the COVID-19 pandemic.

There is extensive literature that stipulates processes in the development of occupational burnout in dentistry. ${ }^{3}$ One theoretical construct that has concentrated efforts to understand how staff are affected in direct service contact with patients is emotional exhaustion. ${ }^{4}$ Although much has been written on assessing and reporting levels of emotional exhaustion in various clinical groups (eg, dental students, ${ }^{5}$ dental hygienists, ${ }^{6}$ dentists ${ }^{7}$ ), there is less information available on the course of this experienced fatigue over time. The field of occupational stress and burnout is dominated by cross-sectional survey designs. ${ }^{8}$ A rare longitudinal study on a representative sample of Dutch dentists presented some evidence that emotional exhaustion preceded the other two dimensions of burnout namely: depersonalisation and personal accomplishment. ${ }^{9}$ A further study of $71 \%$ of Finnish dentists ${ }^{10}$ showed that burnout and depression had reciprocal effects on each other, somewhat contradicting the Dutch study findings. Interestingly, the design of both studies included two waves of data collection spaced approximately 3 years apart which enabled the investigators to claim some causal direction in their findings. However, even two time points present some difficulty in specifying the dynamic nature of the burnout processes and related variables. For example, it is likely that some staff included in the study will respond more quickly to their job environment and conditions than others. In the period of 3 years, it could be argued that staff may have changed and adapted to return to their original level. An earlier study by the same Dutch group ${ }^{11}$ of 50 newly trained dentists successfully followed up a proportion of the original sample 6 years later. Interestingly, they found no significant changes in emotional exhaustion in this extensive period. Again, consideration of the possible rise and fall, that is, within-subject variation, during the early career period of these staff was notably absent. The position taken most frequently in the field of burnout and occupational mental health suggests a fluctuating pattern in response to challenges of working conditions, policy change and public health emergencies. It is notable that these previous sophisticated longitudinal studies presented were not focused on observing personcentred changes associated with a major national health challenge to healthcare services. In addition, an extensive review of stress in dental staff failed to find data that implicated their psychological status to clinical decisionmaking. ${ }^{12}$ More recently, empirical research showed that clinical decision-making was less sensitive in dental staff under time pressure or reporting anxiety. ${ }^{13} 14$ There is increasing recognition that early intervention in newly trained healthcare staff is warranted, ${ }^{15}$ although the evidence base on initiating and designing these educational programmes, to reduce emotional exhaustion, is still very limited. ${ }^{16}$ Furthermore, some unexpected results are found in testing early developments of interventions including effects limited to only a year post-intervention. ${ }^{17}$ Hence, to enable more detailed inspection of the psychological responses of staff to occupational changes, we believe that a further methodological approach is indicated.

The field of intensive longitudinal investigation is gaining acclaim as an important methodological approach to determine, more closely, changes and development of psychological constructs over time and their inter-relationship. ${ }^{18} \mathrm{~A}$ call for future research in the field of burnout has been made to concentrate on 'shortitudinal ${ }^{19}$ as well as longitudinal designs. ${ }^{8}$ The complexity of the conceptual system employed to clarify development of burnout and depression can be better understood by virtue of the frequent assessment of psychological constructs from the same sample with the aid of the theoretical model based on our previous work. We presented a detailed model of how features of burnout relate to depressive symptomatology and other important related issues of preparedness and trauma, in dental staff working in primary care and trainees. ${ }^{2}$ Results from our cross-sectional survey in Scotland provided some support, although we recognise that more research is needed to confirm and develop the conceptual model.

The study reporting on a representative sample from the Netherlands ${ }^{10}$ argued that emotional exhaustion was a precursor of burnout syndrome hence our research group selected, deliberately, this area to focus close attention for further study, especially the development of burnout over time. Our interest was to determine what factors may be responsible for emotional exhaustion developing. The link between depression and emotional exhaustion has been reported either in 'snapshot ${ }^{20}$ or longitudinal studies with lengthy follow-ups of at least a year. ${ }^{21}$ The development of emotional exhaustion over shorter periods of months is of specific interest theoretically to determine if changes are detectable and predictable in the shorter term. For example, in our previous report, ${ }^{2}$ our model highlighted the importance of dental staff being prepared for delivery of their service during the COVID-19 pandemic. In addition, the staff's ratings of trauma experiences and depressive symptomatology were linked to burnout levels. Our model was reconfigured for assessing change over a period of 6 months (figure 1) and states that staff fatigue trajectory may increase or reduce dependent on initial levels of depressive symptomatology controlling for preparedness and trauma experienced. The sign values (+ve or $-\mathrm{ve})$ denote the direction of the proposed pairwise relationships. The job demands-resources (JD-R) model of burnout development states that low resources and high demands result in staff experiencing burnout. The COVID-19 pandemic in 


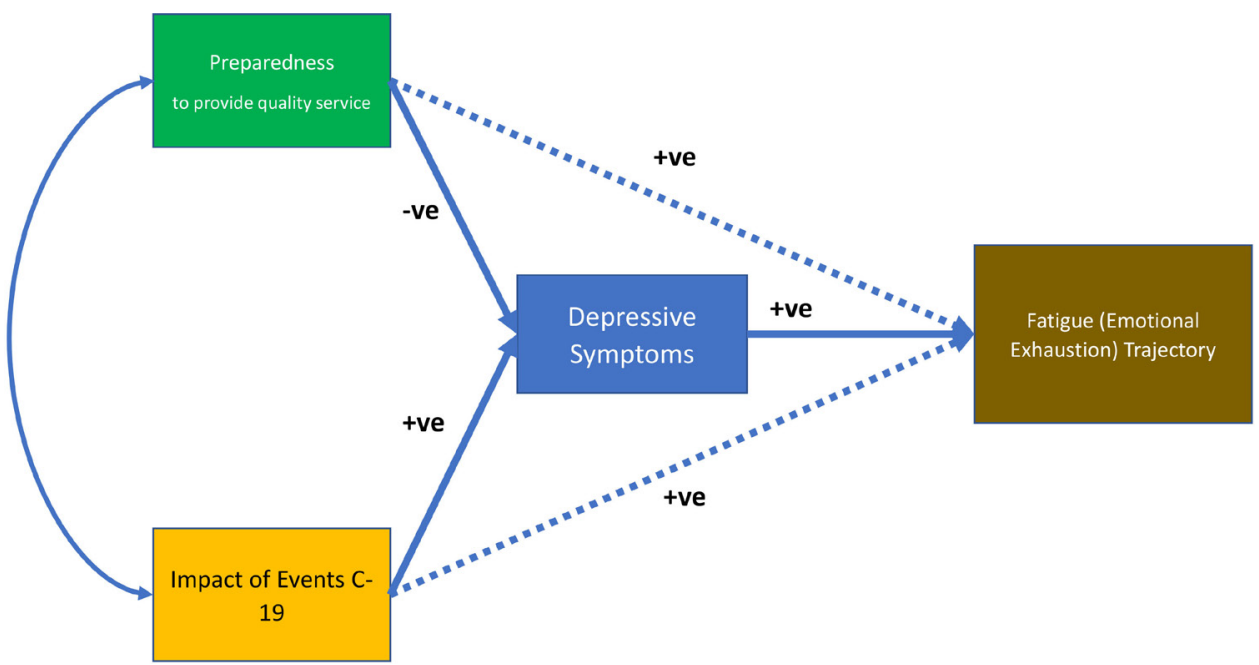

Figure 1 Proposed theoretical model including hypothesised valence of each substantive association of predictor variables to explain emotional fatigue trajectory. Solid straight lines denote pathways from exogenous variables to endogenous variable: depression and onto outcome dependent variable. Dotted lines denote direct effect of exogenous variables onto fatigue trajectory. Curved line with double-headed arrows represents correlation, hence the effects of each endogenous variable are independent of the other.

the second half of 2020 in Scotland was marked by threats of increased national restriction and lockdown. ${ }^{22}$ In terms of service remobilisation, some flexibility of care was introduced from 22 June 2020 with dental practices able to see National Health Service (NHS) patients for faceto-face consultation in need of urgent care, using nonaerosol-generating procedures, and from 13 July 2020 for non-aerosol routine care. In the latter half of 2020 , dentistry moved into 'phase 4' of the NHS Dental Remobilisation Plan where on 1 November contractors could 'provide the full range of NHS treatments to all patients in need of both urgent and non-urgent care'. ${ }^{23}$ Simultaneously, the current position at the time showed no direct relationship between dental treatment or surgery and possible COVID-19 transmission. ${ }^{24}$ Subsequently, however, evidence was reported to show increased risk of exposure and transmission of COVID-19 in the dental care workforce compared with other occupations. ${ }^{25}$

Hence, high demands were transferred to staff by preparations to maintain a quality service where possible and some staff suffered depleted resources both internal and external from experiences of the impact of COVID-19 events and associated low mood.

The results of this new proposed investigation will supply much needed evidence and encourage managers to intervene in a timelier fashion, that is, within weeks rather than years. Hence, the aim of this research was to investigate the variability and development of emotional exhaustion over an extended period of months during the COVID-19 pandemic for dentists and dental health professionals in primary dental care, and for those in training, using an intensive longitudinal design. Three research questions (RQs) were formulated, namely:

RQ1: What was the estimated overall fatigue trajectory over the 6-month period of study?
RQ2: How variable was this fatigue trajectory and can evidence confirm a significant mix of participants that gain or reduce fatigue over time?

RQ3: If there was significant variability in fatigue trajectory, can this be explained by baseline depressive symptomatology controlling for levels of preparedness and trauma response to the COVID-19 pandemic?

\section{METHODOLOGY \\ Participant involvement}

Prior to the design of the study, a series of group sessions with a volunteer sample of trainee dentists (see online supplemental file for definitions) from Scotland ( $\mathrm{N}=12)$ was conducted by two of the authors (RF and GH; trained mental health specialists) during February-April 2020. The topic of these joint sessions was to exchange among the participants the psychological effects of the COVID-19 pandemic. From these sessions, which were primarily of a supportive and therapeutic nature for the volunteers, the current key issues of training preparedness, trauma, depressive symptoms and emotional exhaustion were raised, discussed and views exchanged. The dynamic quality of these psychological reports that were shared frequently among group members, especially the cumulative nature of the uncertainty of the future because of the COVID-19 pandemic both professionally and pragmatically in delivery of patient services, confirmed to the research team the major conceptual structure and RQs.

\section{Baseline survey}

Sample

The baseline survey was open for trainees to respond over a period of 6 weeks from mid-June to end of July 2020 (see online supplemental file for summary details of 
cross-sectional survey sample). By August 2020, dentists and dental health professionals in primary dental care settings in Scotland, who had an NHS Education for Scotland (NES) Portal account (and had opted to receive marketing communications) were also notified of the opportunity to participate. The baseline survey was open for dentists and dental health professionals in primary dental care settings to respond from mid-August to early October 2020.

\section{Outcome measures}

The baseline survey sections comprised the following psychometric measures.

The Patient Health Questionnaire-2 is a two-item screening questionnaire used to assess depressive symptomatology. ${ }^{26}$ The stem of the scale was: 'Over the last 2 weeks, how often have you been bothered by any of the following problems?' The items were worded: 'little interest or pleasure in doing things' and 'feeling down, depressed or hopeless' on an answering scale: 'not at all' (1), 'several days' (2), 'more than half the days' (3) and 'nearly every day' (4).

Dental Professional Preparedness for Practice Scale (DPPPS) consisted of 14 items that were derived from the original designed for medical service personnel. ${ }^{27}$ A full description of the items and factorial structure is provided in our previous publication. ${ }^{2}$ The subscale of seven items was named: P-Qual-C19. It was created from the 14 items of the DPPPS which had the stem: 'In the current COVID-19 pandemic, how well are you prepared for?' Respondents checked one rating from the five available categories: 'unprepared' (1), 'not well prepared' (2), 'prepared' (3), 'well prepared' (4), 'extremely well prepared' (5). The subscale, devised by the authors, was divided by the number of items comprising the subscale to derive a score ranging from 1 to 5 . The internal consistency (Cronbach's alpha) derived from the cross-sectional study was 0.879 .

Impact of Event Scale-Revised comprises of 22 items with three subscales to assess intrusions (8 items), avoidance ( 8 items) and hyperarousal ( 6 items). This validated measure describes the three major components of posttraumatic stress disorder. ${ }^{28}$ The wording of the scale was identical with the addition "with reference to the COVID-19 pandemic and effects to your training or on your workplace'.

Finally, the questionnaire enquired of the respondent's dental professional group, service area, type of practice (where applicable), redeployment status and demographics (age, sex).

\section{Diary questionnaire}

Sample size

In order to test the addition of a predictor variable (depressive symptomatology) to be associated with fatigue trajectory at a standardised level of at least 0.15 via a path model using multiple regression with two control covariates (trauma and preparedness), the sample size required would be 47 participants with an alpha of 0.05 and power of $80 \%$ (using the power pcorr procedure in Stata V.15).

\section{Outcome measure}

The diary questionnaire consisted of three self-report items from the emotional exhaustion Maslach Burnout Inventory (MBI) subscale. Respondents were asked to rate themselves on how they felt at each weekly report. The items were worded: 'I feel emotionally drained from my work', 'I feel used up at the end of the day' and 'I feel burnt out from my work'. A simplified answering scheme of five categories was employed for each item: "not at all' (scored 1), 'a little bit' (2), 'moderately (3), 'quite a bit' (4), 'extremely' (5). A total score was computed by summing all three items to produce a scale ranging from 3 to 15 .

\section{Administration of the baseline survey and diary questionnaires}

The baseline survey was distributed electronically to all participants. Invitations were sent to email addresses associated with the NES Portal accounts of those in the primary care setting who had selected a relevant professional group upon their registration with Portal. Portal is an online tool used for course bookings/management administered by NES. The trainees were invited to take part through their relevant training hub, with support from the NES Postgraduate Dental Dean.

The covering email for the CAREER baseline survey advised participants that if they took part they would also have the opportunity to give their views in more detail by completing a brief weekly diary for a 6 -month period.

Consent was obtained for those interested in volunteering to keep a weekly diary related to their emotional state and routine via an online informed consent form, which all participants in the CAREER Study were required to complete in advance of answering the baseline questionnaire. Diary volunteers were then contacted by email with a link to access the online diary form made available on the survey platform Questback. The form asked participants to select their response to each of three items drawn from the emotional exhaustion subscale of the MBI and then in the open text box describe any significant issues or concerns they had experienced during the preceding week because of the impact of the COVID-19 pandemic on their work or training. The research group asked participants to try and complete their diary entry every Friday, or as near as possible to the close of each working week, while events were still fresh in their minds.

The research administrator reviewed diary completion rates at the beginning of each working week. Where entries for the preceding week were complete, participants were sent a thank you email with the link to make their next diary entry. Where entries for the preceding week were outstanding, participants were sent a personalised reminder email to encourage them to complete their entry as soon as possible. The research group agreed that in cases where three reminders had been sent yet no 
further diary entry had been made, then the reminders would be discontinued and the participant not contacted again unless they recommenced completing their diary independently.

\section{Statistical analysis}

The weekly diary responses were maintained on Questback and were only directly accessible to the research administrator until the final entry was made at which point the full data set was downloaded for analysis. Another member of the research group attached a participant identification number to each diary entry so that the data were pseudonymised for analysis and entries could be cross-referenced to one another and to the baseline survey data. The pseudonymised data were then shared with the chief investigators via a secure server for analysis purposes.

Hence, a record of fatigue ratings was compiled over the duration of the data collection period. The data were transformed into a 'long format' file with the time stamp of the completion included and a summated total $\left(x_{i j}\right)$ of the three items collated to produce a scale score of range $3-15$, where 15 would be considered the maximum fatigue rating.

A growth curve analysis was performed (mixed procedure in STATA V.15) ${ }^{29}$ to derive predicted linear trajectories (ie, slopes) specifying a multilevel maximum likelihood model. ${ }^{30}$ The model is specified in two levels: 1 and 2. The random coefficient model using the notation of Raudenbush and Bryk (as recommended ${ }^{31}$ ) specifies a level 1 model: $y_{i j}=\beta_{0 j}+\beta_{1 \times j_{i j}}+r_{i j}$, where the intercept $\beta 0_{j}$ and slope $\beta 1_{j}$ are individual staff coefficients. Their level 2 models have these coefficients as responses:

$\beta_{0 j}=\gamma_{00}+v_{0 j}$ (intercepts as outcomes)

$\beta_{1 j=} \gamma_{10}+v_{1 j}$ (slopes as outcomes)

This two-level linear model was estimated with random intercepts and slopes. ${ }^{32}$ Variance statistics were interpreted. ${ }^{33}$ Estimates were file stored by participant identification number for further analysis. This file was merged with the baseline survey data reported previously. ${ }^{2}$ The distributional properties of the derived slopes and predictor variables (mental health, trauma and preparedness) were inspected to check assumptions. Path analysis ${ }^{34}$ was performed (sem procedure in STATA V.15), adopting the a priori model in figure 1 , to explain the trajectory variance in the diary volunteer respondents. ${ }^{35}$ Residuals were inspected to assess model fit and additional model specifications were attempted to aid interpretation. Twosided alpha was 0.05 .

\section{RESULTS}

The number who volunteered to participate in the diary study was 62 , with 53 supplying valid data of a minimum of 4 weekly reports over the period from July to December. The mean levels of the psychological variables, namely preparedness and burnout subscales, total Impact of Event Scale and depressive symptomatology of this subsample were no different to the main baseline survey already reported (all $\mathrm{p}>0.1$ ). The maximum number of weekly reports was 24 , and the average was 11 . The three fatigue items were completed without missing ratings from any submission. Individual item frequencies showed that $8 \%$ of weekly submissions (pooled) showed extreme ratings for being emotionally exhausted or burnt out by their work. Ten per cent of weekly reports indicated extreme values for being 'used up by the end of the day' (table 1 ). The average total score was 7.19 (median $=6$, 25th percentile $=4$ and the 75 th percentile $=10$ and $\mathrm{SD}=3.69$ ). The internal consistency was high, demonstrating a Cronbach's alpha: 0.95 , with an average interitem correlation of 0.95 .

A mixed linear growth model was constructed with fatigue total scores as the dependent variable and weekly diaries time stamped from the baseline week in the first week of July (coded zero). Subsequent weeks were coded in units $(1,2,3 \ldots$ etc $)$ to the maximum. Each respondent acted as a cluster in the multilevel model and the estimates of intercepts and slope (fixed effects) and the random effects are presented in table 2, and the plotted values (figure 2) for the slope in the form of a histogram (upper panel) and 'spaghetti' plot (lower panel). The results were estimated without warnings from the statistical routine and converged in three iterations. Both robust and raw SEs were estimated and revealed no substantive differences in interpretation. The raw SEs were employed which enabled both the Wald and likelihhood ratio (LR) tests of overall variance explained to be computed. Both tests were highly statistically significant (Wald $\mathrm{X}^{2} 16.13, \mathrm{df}=1, \mathrm{p}=0.001$; LR test vs the conventional linear model, $\left.\mathrm{X}^{2}=673.26, \mathrm{df}=3, \mathrm{p}<0.001\right)$. Inspection of

\begin{tabular}{llllll}
\hline \multicolumn{2}{l}{ Table 1} & \multicolumn{4}{l}{ Means (SDs) and aggregate frequencies for the fatigue items and total score } \\
\hline & Item & Mean (SD) & Low \%* & Medium \% & High \% (of which extreme \%) \\
\hline 1 & I feel emotionally drained from my work & $2.48(1.08)$ & 58.9 & 17.3 & $24.0(8)$ \\
2 & I feel used up at the end of the day & $2.48(1.12)$ & 58.5 & 16.9 & $24.5(10)$ \\
3 & I feel burnt out from my work & $2.24(1.19)$ & 64.7 & 14.6 & $20.7(8)$ \\
Total & Fatigue scale & $7.18(3.69)$ & & &
\end{tabular}

*Low: combined categories 'not at all' and 'a little'; medium: 'moderately'; high: combined categories 'quite a lot' and 'extremely'. Extremely respondents expressed as percentage (in brackets). 
Table 2 Parameter estimates for linear growth model of fatigue

\begin{tabular}{|c|c|c|c|c|c|c|}
\hline & \multirow[b]{2}{*}{ Est } & \multirow[b]{2}{*}{ SE } & \multirow[b]{2}{*}{$\mathbf{z}$} & \multirow[b]{2}{*}{ P value* } & \multicolumn{2}{|l|}{$95 \% \mathrm{Cl}$} \\
\hline & & & & & Lower & Upper \\
\hline \multicolumn{7}{|l|}{ Fixed effects (intercept, slopes) } \\
\hline Time (weeks)† & 0.13 & 0.03 & 4.02 & $<0.001$ & 0.07 & 0.19 \\
\hline \multicolumn{7}{|c|}{ Random effects ((co-)variances) } \\
\hline Intercept & 12.61 & 3.36 & 3.69 & 0.0003 & 7.48 & 21.25 \\
\hline Time (weeks) & 0.038 & 0.011 & 3.37 & 0.0008 & 0.021 & 0.068 \\
\hline Intercept and time & -0.38 & 0.18 & 2.30 & 0.021 & -0.71 & -0.06 \\
\hline \multicolumn{7}{|l|}{ Level 1 (within person) } \\
\hline Residual & 2.76 & 0.18 & 15.65 & $<0.001$ & 2.43 & 3.13 \\
\hline
\end{tabular}

$\mathrm{N}=53$. Wald $\mathrm{X}^{2}(\mathrm{df} 1)=16.13, \mathrm{p}=0.0001$, LR test vs linear model $\mathrm{X}^{2} 673.26, \mathrm{p}<0.0001$.

${ }^{*}$ All $p$ values are two tailed with exception of variances (conventional one tailed).

†Time is coded $0=$ week 1 , with equal intervals of unit 1 for each additional week, max 24 weeks.
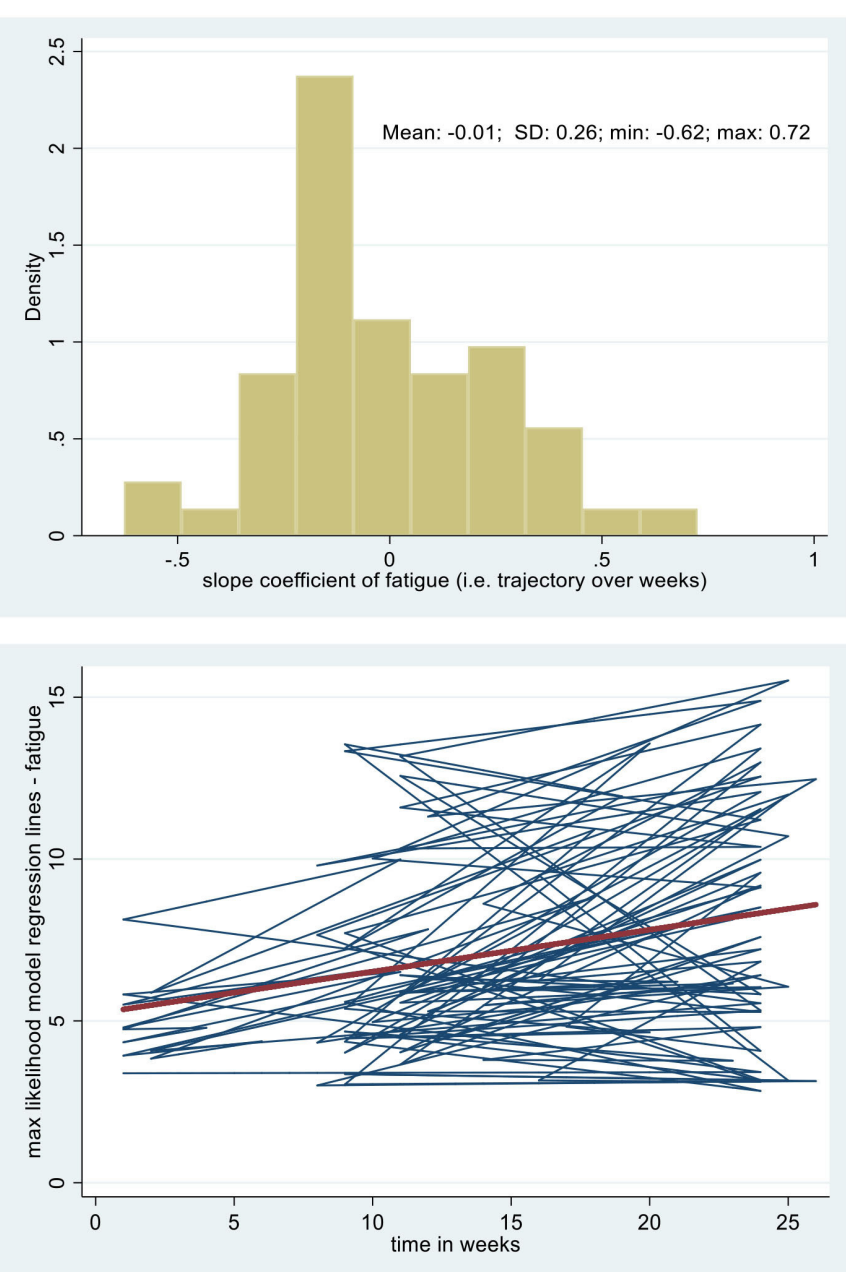

Figure 2 Histogram of slopes from model-based predictions of fatigue as a function of week during COVID-19 pandemic (July-December 2020) with mean and SD presented (upper panel). Spaghetti plot of sample average (thick) and individual staff-specific regression lines (lower panel). Note different starting points for some staff. table 2 indicates that the average baseline fatigue score for the respondents was 5.36 (95\% CI: 4.27 to 6.45 ) and the average slope, that is the trajectory, was 0.13 (95\% CI: 0.07 to 0.19 ). So that over a 10 -week period, the average respondent would increase their total fatigue score by 1.3 scale units. Over the 24-week period, it would be expected that respondents would select approximately a category higher, on average, for each of the three items from their initial baseline rating. The trajectories of the respondents varied significantly $(\mathrm{p}=0.001)$ as shown in figure 2 (upper and lower panels). Calculating the slope variance as an SD (ie, square root of 0.038 ) which equals 0.19 , it can be estimated that $95 \%$ of the respondents vary between \pm 0.39 units of the typical slope. The between-persons variability in the predicted slopes in figure 3 appears to match these numbers.

Merging the baseline survey data with the diary questionnaire data resulted in 48 full data sets that consisted of the fatigue trajectory summary estimate for each staff member, their baseline trauma, preparedness and mental health baseline scores and some demograghics. The mean age of this diary respondent sample was 42.2 years ( $\mathrm{SD}=12.8$, range $22-62)$ and $77 \%$ were female. Initial mean levels of the fatigue trajectories were inspected across gender and trainee/primary care staff categories ( $\mathrm{t}$ values returned $\mathrm{p}>0.7)$. The correlation between age and trajectory was trivial $(\mathrm{r}=-0.13, \mathrm{p}>0.4)$. The starting point when respondents uploaded their results and the number of weekly submissions per respondent were unrelated to the fatigue trajectory slope, $\mathrm{r}=-0.02$ and $\mathrm{r}=0.03$, respectively (both $\mathrm{p}>0.8$ ). The level of self-rated clinical work under NHS auspices on a 5-point scale ranging from 'none' to 'mostly NHS' was not correlated with the sample trajectories $(r=0.09, p>0.5)$. These slopes conformed to a normal distribution (skewness and kurtosis test for normality, $\left.\mathrm{X}^{2}=2.91, \mathrm{p}>0.23\right) .{ }^{36}$ The data from this merged file were fitted to the theoretical model presented previously 


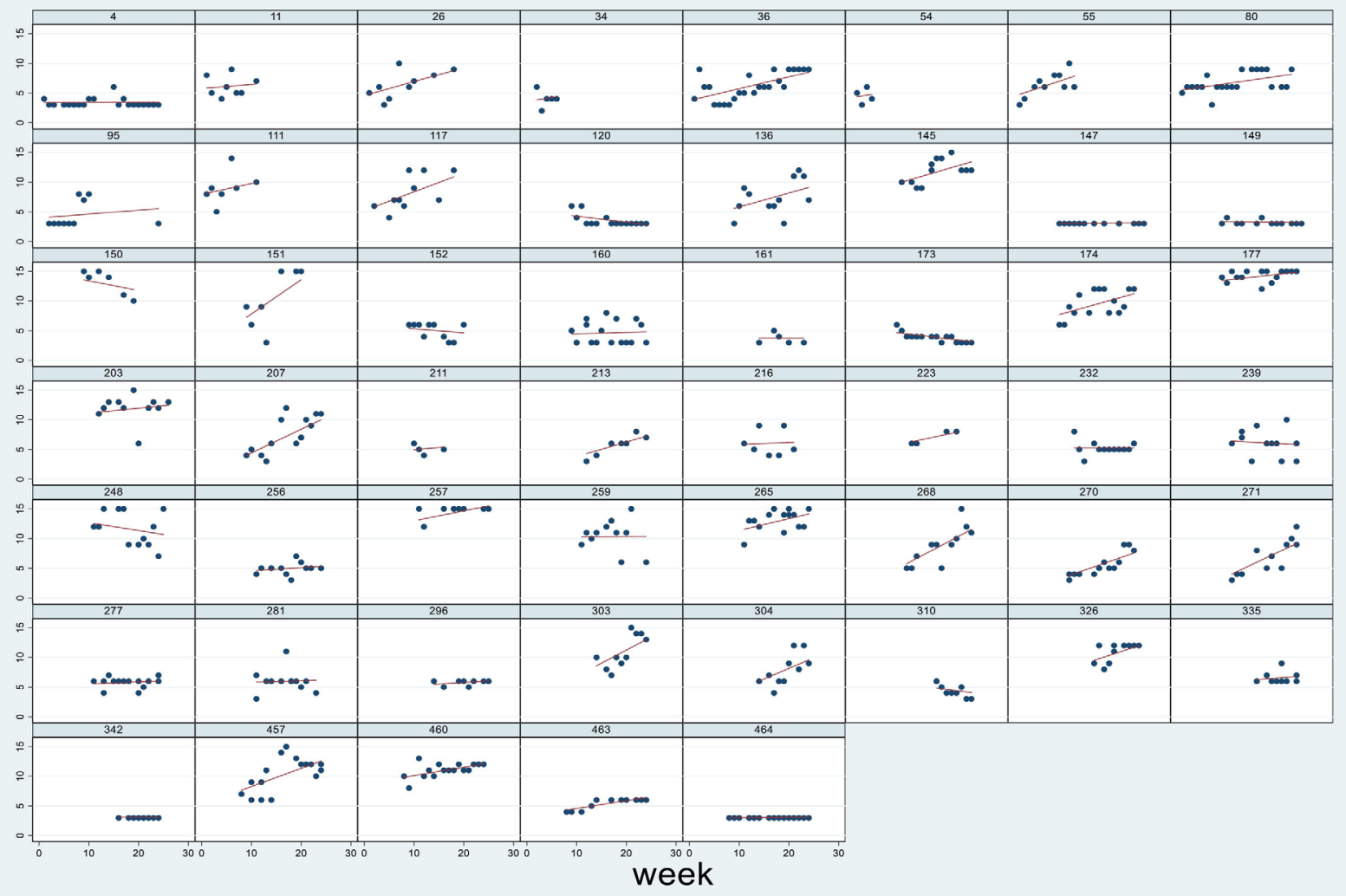

Figure 3 Panel plots (each plot constitutes a single respondent) of raw fatigue data (total scale score of three-item ratings: min: 3, max: 15) collected weekly over July-December 2020 of the COVID-19 pandemic and model-predicted regression lines (red) for 53 staff.

(figure 1) and converged easily with a maximum likelihood estimator. The standardised results are summarised in figure 4 as a path diagram. The endogenous variable (depressive symptomatology) was negatively associated with the fatigue trajectory. The exogenous variables (preparedness and trauma) were positively associated with individual slopes of fatigue. These latter two associations were found to be of very similar magnitude (LR

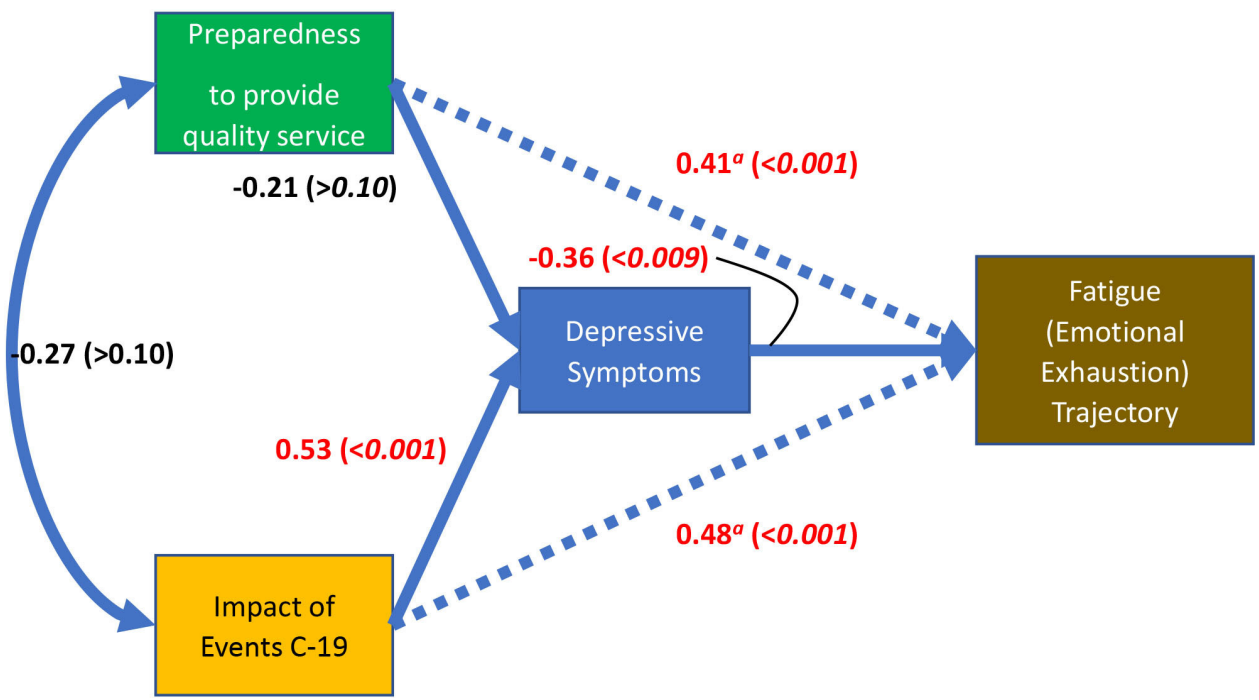

Figure 4 Path analysis of raw data $(\mathrm{N}=48)$ applied to the theoretical model with fatigue trajectories as the dependent variable, depressive symptoms (endogenous variable) and the exogenous variables: preparedness and trauma, using same diagrammatic formatting for the representation of paths. Residual error terms omitted to improve clarity. Standardised coefficients with $\mathrm{p}$ values in brackets. Significant parameter estimates in red font. Superscript symbol a denotes approximate identical parameter values $(p>0.5)$. 
test $\left.X^{2}=0.39, d f=1, p>0.5\right)$. The belief of being prepared to provide a quality service and the psychological trauma level reported at the start of the diary data collection were predictive, on average, of increasing fatigue over the course of the study. Respondents rating themselves as experiencing depression symptoms, controlling for preparedness and trauma levels, tended to reduce their fatigue trajectory, that is, those respondents who reported more depressive features at 'baseline' were less likely to report an increase in their emotional fatigue over time. The model fit was reasonable $\left(\mathrm{R}^{2}=0.56\right)^{37}$ and the residuals were of low value (Standardised Root Mean Square Residual $=0.03$ ).

\section{DISCUSSION}

The main aim of this study was to investigate the course of emotional exhaustion over approximately a period of 6 months that coincided with a gradual increase in restrictions from Scottish Government as a result of increased COVID-19 cases $^{22}$ Each RQ will be addressed in turn.

RQ1-fatigue ratings increased steadily, on average, over the period of the weekly diary returns. The likely increase in emotional exhaustion was found to be approximately three units of the fatigue scale that ranged from 3 to 15 over the 24-week study duration. That is, the average rating can be considered to be about $25 \%$ greater at the end of the study period compared with the start.

RQ2- a focus on the average staff respondent was of interest however this masks, we consider, a central theme of our investigation, namely, the importance of betweensubject variance. There was very significant variation between respondents in their fatigue trajectories. This variation was far from trivial supporting the view that staff differed strongly in their ability to manage emotional demands of treating patients under the constraints of the COVID-19 pandemic. Thus, it is arguable as to whether there is practical usefulness in estimating the average increase of emotional exhaustion when observing respondents showing such a wide range of trajectories. We were fortunate in having available the initial baseline crosssectional data to merge with the fatigue trajectories. This enabled an application of our theoretical model to assist answering our final question.

RQ3-the variation in emotional exhaustion trajectory was explained, not by respondents' demographics (age, gender, professional group or training status) but significantly with reference to the respondents' baseline ratings of depressive symptoms controlling for preparedness and COVID-19 trauma. Of interest was the finding that those who were already experiencing depressive symptoms were less prone to developing emotional exhaustion. This effect may demonstrate a blunting of affect over the remaining study period in those already with depressed mood. It is interesting that Seidler $e t a l$, in their extensive systematic review, found convincingly the importance of high job demands being responsible largely for increasing emotional exhaustion in employees. ${ }^{38}$ It may be that those with depressive symptoms simply resist feeling pressure through processes such as learnt helplessness, and therefore emotional exhaustion fails to increase further. It will be noted that this significant effect is the opposite to that hypothesised in our proposed model and hence worthy of further attention.

Other components of the model deserve comment. Those respondents who stated that they were prepared to commit to delivering high-quality dental services were more likely to show increased emotional fatigue over time as predicted. We expected that the high demands of patients requesting service and professional practice in the climate of restrictions (eg, fallow periods between patients and the wearing of physically restrictive personal protective equipment) would be perceived as potential extra burdens. This important effect was paralleled closely by the level of trauma experienced at baseline of the COVID-19 pandemic in mid-2020. ${ }^{2}$ The impact felt from wider events to the public (eg, increases in hospitalisation and death rates), as well as individual experiences of trauma resulting from the pandemic, can be considered to lower the psychological resources of staff. This set of factors summarised under high demands (prepared to deliver quality service) and low resources (due to negative events) parallels the framework proposed by Hakanen and Bakker to explain burnout development. ${ }^{8}$ Respondents therefore were more likely to develop emotional fatigue, reflected in their repeated diary ratings, if they believed themselves more prepared to provide high standards of service, that is, experienced as high demand, and had been influenced more traumatically (that is, reducing personal resources) by events of the COVID-19 pandemic. The current results would therefore support some of the components of the JD-R theory of burnout development referred to previously and that of Hakanen and Bakker's life course approach to burnout.

The relationship between burnout and depression during the COVID-19 pandemic ${ }^{39}$ has shown there is considerable overlap between burnout and depression especially at the end-stage of burnout which mimics depressive state ${ }^{40}$ and is consistent with the work already quoted by Ahola and Hakanen in their Finnish dentist study. ${ }^{10}$ There is some interesting new research which supports the link between vagally mediated heart rate variability (autonomic dysregulation) as a response to stressors and both burnout and depression. ${ }^{41}$ The finding that autonomic dysregulation is an individual quality that relates to both higher levels of burnout and depression is consistent with the view that the two psychological constructs are inextricably linked. In addition, there is a further important theoretical concept that can be raised. It is vital to understand the aggregate levels of burnout and depression with reference to the wider occupational system that the respondents are working within. However, the physiological study mentioned and also additional work by Bianchi et al remind researchers not to dispel the individual variability of burnout and depression. ${ }^{42}$ 
What are the consequences for employers and public bodies of this investigation into fatigue trajectories? An inspection of related literature may assist interpretation. The longitudinal survey of Finnish white-collar professionals consisting of five waves of data collection approximately at 2-year intervals found that emotional exhaustion was a significant precursor of burnout syndrome. ${ }^{43}$ Our findings are resonant and support the 'process model' of Leiter and Maslach ${ }^{44}$ over the phase model. ${ }^{45} \mathrm{~A}$ helpful framework formulated by Almén argues that 'clinical burnout' possesses features, such as the extended experience of burnout and sick leave, that are self-maintaining, thereby strengthening calls for preventive action by employers and management teams. ${ }^{46}$ A large 2-year longitudinal study from Sweden has shown that those reporting a high level of support based on social capital and decision latitude were, surprisingly, not featured in a lower burnout response at follow-up. ${ }^{47}$ The predictors of burnout were initial burnout level and, in particular, work demands. Hence, improving support alone may not be the panacea to reducing susceptibility to burnout, rather employing authorities may be better advised to increasing their sensitivity to managing workload through managerial action to improve good psychological functioning.

This is the first intensive longitudinal study of emotional exhaustion using diaries that we are aware of in the field of occupational health in dental staff. We believe that this occupational group have been more prone to the pressures from the COVID-19 pandemic due to the nature of direct patient contact in service delivery. There are several limitations to this study. First, the collection of diaries was limited. We relied on volunteers who participated originally in the initial crosssectional baseline survey. Although checks were made to show that the subsample who participated in the diary study were not significantly different from the main survey, there may have been differences we were not aware of, hence limiting the degree of generalisability. Likewise, the diary submissions were incomplete over the course of the data collection period and estimates of the trajectories were based, in some cases, on as few as four data points. A second major limitation we recognise was that the model we present was tested using raw data variables, with measurement error hence attenuating the associations presented. Our sample size was limited. This prevented latent variable expression of the major constructs. The model itself is one of a number that could have been tested even though we based the presented framework on a version of our previous theoretical understanding. However, with these limitations in mind, we were encouraged by the enthusiasm shown by our respondents who uploaded their ratings without any practical incentive over the course of the study. Furthermore, this research, we believe has identified a fuller explanation of the dynamics of an important component believed to be a crucial precursor to longer term occupational suffering, namely burnout.

\section{FUTURE WORK}

There is a burgeoning need to develop ways of intervening to prevent emotional exhaustion and depressive symptomatology as has been raised already. It is recognised that reducing job demands might be an easily measurable method to protect staff and further investigation is urgently needed to confirm these recent findings. ${ }^{47}$ The fact that many staff will have been adversely affected both professionally and personally over the pandemic requires investigators to assess the recovery of staff. Recently, five different profiles have been identified of staff that adopt different strategies of recovery and this work could be important to expand within the field of dentistry to examine the features of these various groups and the methods they as individuals employ to maintain their psychological health. ${ }^{48}$ The high degree of uncertainty experienced by dental practices and their teams within the UK during the period of July-December $2020^{49}$ has necessitated flexibility of response. The likelihood is that further changes in practice, over the years ahead because of the COVID-19 experience, will be necessary. Such changes will give researchers opportunity to observe the behaviour of staff and their psychological reactions. The use of intensive longitudinal methodology has much to recommend charting these responses and enable researchers to work alongside staff and employers to improve working conditions and psychological well-being.

Twitter Mariana Araujo @AraujoMarianaP, Siyang Yuan @SIG_Yuan and Gerald Humphris@GHumphris

Acknowledgements We gratefully acknowledge the assistance of Lorna Barnsley (administrator for this project), NHS Education for Scotland for the resources from their team and use of their Portal system and Questback license, and all the participants who completed the baseline survey. We are especially grateful to the volunteers who completed the diary questionnaires, specifically for their time, effort and openness during such unprecedented and challenging times.

Contributors Initial panel forum with trainee group-RF and GH. Conceptual design of study—RF, GH, JK, LY and JC. Questionnaire design and preparation of material for online use by potential participants-JK, LB, SY and MA. Initial manuscript preparation-RF and GH. Statistical analysis-GH. Sample recruitment liaison with NHS Education Scotland-JK, LY and JC. Draft manuscript preparation, comment and feedback—all authors. Research team planning —all authors. Manuscript reading and approval—all authors. GH responsible for the overall content as guarantor.

Funding The authors have not declared a specific grant for this research from any funding agency in the public, commercial or not-for-profit sectors.

Competing interests All members of the CAREER Project team, comprising the authors, were required to declare any financial, intellectual and other relevant interests at the outset of the project. None were declared nor did any arise during the course of the project.

Patient consent for publication Obtained.

Ethics approval This study involves human participants and was approved (18 May 2020) by the University of Dundee Nursing and Health Sciences and Dentistry Research and Ethics Committee (reference: UODISDENISTAFFI20201013-Freeman). A participant information sheet containing detailed information about the study was provided, and written consent was collected from all participants.

Provenance and peer review Not commissioned; externally peer reviewed.

Data availability statement Data may be obtained from a third party and are not publicly available. Raw data held by NHS Education Scotland and all personal data collected were managed in line with General Data Protection Regulations (GDPR) 2018. For more information, please see the NES Privacy Policy document (http:// www.nes.scot.nhs.uk/privacy-and-data-protection.aspx).

Supplemental material This content has been supplied by the author(s). It has not been vetted by BMJ Publishing Group Limited (BMJ) and may not have been peer-reviewed. Any opinions or recommendations discussed are solely those of the author(s) and are not endorsed by BMJ. BMJ disclaims all liability and 
responsibility arising from any reliance placed on the content. Where the content includes any translated material, BMJ does not warrant the accuracy and reliability of the translations (including but not limited to local regulations, clinical guidelines, terminology, drug names and drug dosages), and is not responsible for any error and/or omissions arising from translation and adaptation or otherwise.

Open access This is an open access article distributed in accordance with the Creative Commons Attribution Non Commercial (CC BY-NC 4.0) license, which permits others to distribute, remix, adapt, build upon this work non-commercially, and license their derivative works on different terms, provided the original work is properly cited, appropriate credit is given, any changes made indicated, and the use is non-commercial. See: http://creativecommons.org/licenses/by-nc/4.0/.

Author note This paper is dedicated in the memory of our colleague and friend Professor Ruth Freeman.

ORCID iD

Gerald Humphris http://orcid.org/0000-0002-4601-8834

\section{REFERENCES}

1 Sanghera J, Pattani N, Hashmi Y, et al. The impact of SARS-CoV-2 on the mental health of healthcare workers in a hospital setting-A systematic review. J Occup Health 2020;62:e12175.

2 Humphris G, Knights J, Beaton L, et al. Exploring the effect of the COVID-19 pandemic on the dental team: preparedness, psychological impacts and emotional reactions. Front Oral Health 2021;2.

3 Mohebbi SZ, Gholami M, Chegini M, et al. Impact of career choice motivation on academic burnout in senior dental students: a crosssectional study. BMC Med Educ 2021;21:52.

4 Slabšinskienè E, Gorelik A, Vasiliauskienè I, et al. Factorial validity and variance of the Maslach burnout inventory between demographic and workload groups among dentists of Lithuania. Int $J$ Environ Res Public Health 2020;17 doi:10.3390/ijerph17249154

5 Gorter R, Freeman R, Hammen S, et al. Psychological stress and health in undergraduate dental students: fifth year outcomes compared with first year baseline results from five European dental schools. Eur J Dent Educ 2008;12:61-8.

6 Patel BM, Boyd LD, Vineyard J, et al. Job satisfaction, burnout, and intention to leave among dental Hygienists in clinical practice. J Dent Hyg 2021;95:28-35.

7 Molina-Hernández J, Fernández-Estevan L, Montero J, et al. Work environment, job satisfaction and burnout among Spanish dentists: a cross-sectional study. BMC Oral Health 2021;21:156.

8 Hakanen JJ, Bakker AB. Born and bred to burn out: a life-course view and reflections on job burnout. J Occup Health Psychol 2017;22:354-64.

9 Te Brake H, Smits N, Wicherts JM, et al. Burnout development among dentists: a longitudinal study. Eur J Oral Sci 2008;116:545-51.

10 Ahola K, Hakanen J. Job strain, burnout, and depressive symptoms: a prospective study among dentists. J Affect Disord 2007;104:103-10.

11 Gorter RC, Storm MK, te Brake JHM, et al. Outcome of career expectancies and early professional burnout among newly qualified dentists. Int Dent J 2007:57:279-85.

12 Plessas A, Delgado MB, Nasser M, et al. Impact of stress on dentists' clinical performance. A systematic review. Community Dent Health 2018;35:9-15.

13 Plessas A, Nasser M, Hanoch $\mathrm{Y}$, et al. Impact of time pressure on dentists' diagnostic performance. J Dent 2019;82:38-44.

14 Chipchase SY, Chapman HR, Bretherton R. A study to explore if dentists' anxiety affects their clinical decision-making. Br Dent $J$ 2017;222:277-90.

15 Chapman HR, Chipchase SY, Bretherton R. The evaluation of a continuing professional development package for primary care dentists designed to reduce stress, build resilience and improve clinical decision-making. Br Dent J 2017;223:261-71.

16 Mikhaiel JP, Pollack J, Buck E, et al. Graduating with Honors in resilience: creating a whole new doctor. Glob Adv Health Med 2020;9:2164956120976356.

17 Brake HT, Gorter R, Hoogstraten J, et al. Burnout intervention among Dutch dentists: long-term effects. Eur J Oral Sci 2001;109:380-7.

18 Bolger. N, and Laurenceau. J-P. Intensive longitudinal methods: an introduction to diary and experience sampling research. New York: The Guilford Press, 2013.
19 Dormann C, Griffin MA. Optimal time lags in panel studies. Psychol Methods 2015;20:489-505.

20 Bianchi R, Schonfeld IS. Burnout is associated with a depressive cognitive style. Pers Individ Dif 2016;100:1-5.

21 Hakanen JJ, Schaufeli WB. Do burnout and work engagement predict depressive symptoms and life satisfaction? A three-wave seven-year prospective study. J Affect Disord 2012;141:415-24.

22 Wikimedia Foundation, Inc. Timeline of the COVID-19 pandemic in Scotland (2020) 2020.

23 Scottish Government. Remobilisation of NHS dental services: phase 4. in: P.C. Directorate, (Ed.). Edinburgh: St Andrews House, 2020: 1-8.

24 Levit M, Levit L. Infection risk of COVID-19 in dentistry remains unknown: a preliminary systematic review. Infect Dis Clin Pract 2021;29:e70-7.

25 Singhal S, Warren C, Hobin E, et al. How often are dental care workers exposed to occupational characteristics that put them at higher risk of exposure and transmission of COVID-19? A comparative analysis. J Can Dent Assoc 2021;87:116.

26 Kroenke K, Spitzer RL, Williams JBW. The patient health questionnaire-2: validity of a two-item depression screener. Med Care 2003;41:1284-92.

27 Barr J, Ogden KJ, Rooney K, et al. Preparedness for practice: the perceptions of graduates of a regional clinical school. Med J Aust 2017;206:447-52.

28 Creamer M, Bell R, Failla S. Psychometric properties of the Impact of Event Scale - Revised. Behav Res Ther 2003;41:1489-96.

29 StataCorp. Stata statistical software: release 15. Texes, USA: StataCorp LLC, College Station, 2017.

30 McNeish D, Matta T. Differentiating between mixed-effects and latent-curve approaches to growth modeling. Behav Res Methods 2018;50:1398-414

31 Nezlek J. Diary methods for social and personality psychology. Los Angeles: Sage, 2012.

32 Rabe-Hesketh S, Skrondal A. Multilevel and longitudinal modeling using Stata, volume I: continuous responses. 3rd edn. Texas: StataCorp, College Station, 2012.

33 Bolger N, Laurenceau JP. Intensive longitudinal methods: an introduction to diary and experience sampling research. Guilford Publications, 2013.

34 Streiner DL. Finding our way: an introduction to path analysis. Can J Psychiatry 2005:50:115-22.

35 Curran PJ, Obeidat K, Losardo D. Twelve frequently asked questions about growth curve modeling. J Cogn Dev 2010;11:121-36.

36 D'Agostino RB, Belanger A, D'Agostino RB. A suggestion for using powerful and informative tests of normality. Am Statist 1990;44:316-21.

37 Cohen J. A power primer. Psychol Bull 1992;112:155-9.

38 Seidler A, Thinschmidt M, Deckert S, et al. The role of psychosocial working conditions on burnout and its core component emotional exhaustion - a systematic review. J Occup Med Toxicol 2014:9:10.

39 Huo L, Zhou Y, Li S, et al. Burnout and its relationship with depressive symptoms in medical staff during the COVID-19 epidemic in China. Front Psychol 2021;12:616369.

40 Bianchi R, Schonfeld IS, Laurent E. Burnout-depression overlap: a review. Clin Psychol Rev 2015;36:28-41.

41 Kanthak MK, Stalder T, Hill LK, et al. Autonomic dysregulation in burnout and depression: evidence for the central role of exhaustion. Scand J Work Environ Health 2017;43:475-84.

42 Bianchi R, Manzano-García G, Rolland J-P. Is burnout primarily linked to Work-Situated factors? A relative weight analytic study. Front Psychol 2020;11:623912.

43 Mäkikangas A, Leiter MP, Kinnunen U, et al. Profiling development of burnout over eight years: relation with job demands and resources. Eur J Work Organ Psychol 2020;5:1-12.

44 Leiter MP, Maslach C. Latent burnout profiles: a new approach to understanding the burnout experience. Burn Res 2016;3:89-100.

45 Golembiewski R, Munzenrider R. Phases of burnout: developments in concepts and applications. Praeger Publishers, 1988.

46 Almén N. A cognitive behavioral model Proposing that clinical burnout may maintain itself. Int J Environ Res Public Health 2021;18 doi:10.3390/ijerph18073446

47 Fagerlind Ståhl A-C, Ståhl C, Smith P. Longitudinal association between psychological demands and burnout for employees experiencing a high versus a low degree of job resources. BMC Public Health 2018;18:915.

48 Siltaloppi M, Kinnunen U, Feldt T, et al. Identifying patterns of recovery experiences and their links to psychological outcomes across one year. Int Arch Occup Environ Health 2011;84:877-88.

49 Westgarth D. COVID-19 and the dental team. BDJ In Practice 2020;33:18-22. doi:10.1038/s41404-020-0585-3 